University of Nebraska - Lincoln

DigitalCommons@University of Nebraska - Lincoln

CSE Conference and Workshop Papers

Computer Science and Engineering, Department

2004

\title{
IMSH: An Iterative Heuristic for SRLG Diverse Routing in WDM Mesh Networks
}

Ajay Todimala

University of Nebraska-Lincoln, ajayt@cse.unl.edu

Byrav Ramamurthy

University of Nebraska-Lincoln, bramamurthy2@unl.edu

Follow this and additional works at: https://digitalcommons.unl.edu/cseconfwork

Part of the Computer Sciences Commons

Todimala, Ajay and Ramamurthy, Byrav, "IMSH: An Iterative Heuristic for SRLG Diverse Routing in WDM Mesh Networks" (2004). CSE Conference and Workshop Papers. 99.

https://digitalcommons.unl.edu/cseconfwork/99

This Article is brought to you for free and open access by the Computer Science and Engineering, Department of at DigitalCommons@University of Nebraska - Lincoln. It has been accepted for inclusion in CSE Conference and Workshop Papers by an authorized administrator of DigitalCommons@University of Nebraska - Lincoln. 


\title{
IMSH: An Iterative Heuristic for SRLG Diverse Routing in WDM Mesh Networks
}

\author{
Ajay Todimala and Byrav Ramamurthy \\ Department of Computer Science and Engineering \\ University of Nebraska-Lincoln \\ Lincoln NE 68588-0115 U.S.A. \\ Email: \{ajayt, byrav\}@cse.unl.edu
}

\begin{abstract}
Survivable routing of a connection involves computation of a pair of diverse routes such that at most one route fails when failures occur in the network topology. A subset of links in the network that share the risk of failure at the same time are said to belong to a Shared Risk Link Group (SRLG) [3]. A network with shared risk link groups defined over its links is an SRLG network. A failure of an SRLG is equivalent to the failure of all the links in the SRLG. For a connection to be survivable in an SRLG network its working and protection paths must be routed on SRLG diverse paths. SRLG diverse routing problem has been proved to be NP-complete in [1]

According to the quality of service requirement of a survivable connection request, dedicated protection or shared protection can be used to establish the connection request. With dedicated protection, the connection is established on both the SRLG diverse working and protection paths. The simplest heuristic for computing SRLG diverse path pair is the two-step approach, but it suffers from the trap topology problem. In [2] an iterative heuristic (ITSH) using the two-step approach was proposed to compute least cost SRLG diverse path pair. Suurballe's algorithm computes a pair of least cost linkdisjoint paths between a node pair. In this work we present a modified Suurballe's heuristic for computing the SRLG diverse routes between a node pair. We then propose an iterative heuristic (IMSH) which uses the modified Suurballe's heuristic for computing the least cost SRLG diverse routes. We also present an 1/2-cost-improvement optimality check criterion for dedicated protection.
\end{abstract}

Keywords: WDM Networks, Shared Risk Link Group (SRLG), Least Cost SRLG Diverse Routing (LC-SDR), Dedicated and Shared Protection.

\section{INTRODUCTION}

WDM networks have gained tremendous popularity due to their ability to tap the enormous amount of bandwidth in an optical fiber. Their growing popularity and bandwidth capacity have made survivability in these networks an important aspect. The physical layer in a WDM optical network consists of nodes inter-connected

This work was supported in part by the U. S. National Science Foundation grants (ANI-0074121 and EPS-0091900). by optical fiber links. The fiber links in the physical layer pass through conduits and right-of-ways. Multiple fiber links may pass through common conduits and right-ofways. When there is a failure in the conduit or right-ofway, all the fiber links passing through the conduit may fail at the same time. Such fiber links that share the same risk of failure are said to be in same shared risk link group (SRLG) [3]. Failure of an SRLG is equivalent to the failure of all the links in the SRLG. SRLG failures can be used to model several types of failure conditions like single-link failures, conduit/right-of-way failures, fiberspan failures, double-link failures or any failure of other possible subset of links sharing a common risk.

The wavelength-level connections in such a network are set up on lightpaths and they can be protected in two ways: dedicated protection and shared protection. In dedicated protection the connection is established on both the working and the protection paths and the data is sent on both the paths. Once the destination detects the failure on the working path it immediately switches to the protection path. In shared protection the connection is established on the working path and the resources are reserved along the protection path. Shared protection allows multiple protection paths to share the resources when their corresponding working paths do not fail at the same time.

Under dedicated protection, the least cost link disjoint path pair problem has polynomial solution [4]. But the problem of least cost link disjoint path pair computation under shared protection is proved to be NP-complete in [5]. In dedicated protection the cost of the working path is independent of the cost of the protection path. We call such cost structure an independent cost structure of dedicated protection. In shared protection, the cost of the protection path depends on the working path. A link can be used by a protection path at no extra cost if it is already being used for protecting some other working path, linkdisjoint from its corresponding working path. We call such a cost structure as dependent cost structure. Due to 
the independent cost structure of dedicated protection, we have a polynomial solution to the least cost link-disjoint path pair problem. In contrast, due to the dependent cost structure of shared protection the problem of least cost link-disjoint path pair is NP-complete [5].

Most of the research on computing least cost SRLG diverse path pair is concentrated on shared protection [2], [5], [6], [7], [8], [9]. In this work we present an iterative heuristic for computing the least cost SRLG diverse path pair for a connection. The iterative heuristic can be used for both shared and dedicated protection. We present an 1/2-cost-improvement optimality verification criterion based on the independent cost structure of dedicated protection.

The rest of the paper is organized as follows. In Section 2 we present the problem formulation. Section 3 presents some of the heuristics for computing the least cost SRLG diverse path pair. In Section 4 we present our iterative modified Suurballe's heuristic (IMSH) for computing least cost SRLG diverse path pair with its optimality verification under dedicated protection. In Section 5 we discuss the experimental results and in Section 6 we give the concluding remarks.

\section{Problem Formulation}

Given a network topology with SRLG constraints and a node pair $(s, d)$ the least cost SRLG diverse routing (LC-SDR) problem is to find a pair of least cost SRLG diverse routes in the physical topology such that at any instant of time at most one of the routes fails due to single SRLG failure. SRLG diverse routing problem is proved to be NP-complete [1]. We assume the independent cost structure of dedicated protection i.e., the cost of using a link for the working path is same as the cost of using the link for the protection path. In this work we consider dynamic traffic but the heuristic proposed can also be used to compute the least cost SRLG diverse paths for static traffic network scenario. The cost function of the edges in the network changes with network state. We assume that all the edges in the graph $G$ have a base cost corresponding to the length of the edge. The cost of the edge increases by a constant factor $\alpha$ with the increasing number of used wavelengths on the edge in addition to the base cost of the edge. The current cost of the edge $(i, j)$, $c(i, j)=b(i, j)+\alpha \times u(i, j)$ where $b(i, j)$ is the base cost of the link $(i, j)$ and $u(i, j)$ is the number of used wavelengths on link $(i, j)$.

\section{RELATED WORK}

There has been considerable research done on computing the SRLG diverse routes on a given topology with SRLG constraints. Many heuristics have been proposed in [10], [11], [12], [2], [13] to compute diverse routes between a given node pair. One of the most formidable problem these heuristics face is the trap topology problem. The trap topology problem is a network scenario where a given heuristic could not find the diverse routes on the given topology between a node pair even though diverse routes exist. It is important to note that the trap topology problem is specific neither to the heuristic nor the network topology but is due to the combination of both. Some heuristics may succeed to find SRLG diverse routes for a given node pair on the given network, while others may fail. In the remainder of this section we will briefly survey previously proposed heuristics.

One of simplest and straight forward method to find a pair of SRLG diverse paths in a given graph $G$ with SRLG constraints is the two-step heuristic (TSH). This approach finds a shortest path $p$ in the graph $G$. It then removes all the links along the shortest path computed and the links that are in SRLG conflict with the links along $p$ in the graph $G$. The approach finds shortest path $p^{\prime}$ in the modified graph $G^{\prime}$. If such a path $p^{\prime}$ exists, then $p$ and $p^{\prime}$ are SRLG diverse in the original graph $G$. In some special cases, however, this approach even fails to find the linkdisjoint paths.

The Iterative Two-Step Heuristic (ITSH) was proposed by P-H. Ho in [2]. The ITSH heuristic executes the twostep heuristic (TSH) iteratively until an optimal solution is obtained. In the $i^{\text {th }}$ iteration it uses $i^{\text {th }}$ shortest path (computed using Yen's $K$ shortest path algorithm [15] or an equivalent algorithm) as the seed path in the TSH. For verification of the optimality the ITSH iterates until the cost of the seed path exceeds the current optimal. A detailed discussion of the ITSH and its optimality verification criterion is presented in [2].

Suurballe's algorithm [4] finds the least cost linkdisjoint path pair in a graph, if such a path-pair exists. The obvious way to solve the problem of least cost link disjoint path pair is to formulate the problem as the network flow problem [14] and find the min-cost maxflow in the network from $s$ to $t$. Suurballe's algorithm essentially uses this idea to give a polynomial algorithm.

Let us briefly discuss Suurballe's algorithm for computing the least cost link-disjoint path pair. Given the directed graph $G=(V, E)$ and the cost function $C$ for the edges in the graph $G$. Initially cost of all the edges is non-negative and $C(i, j)=C(j, i)$. Compute a shortest path $p$ between the node pair $(s, t)$ in graph $G$. Copy the graph $G$ and its cost function $C$ to a modified graph $G^{\prime}$ and cost function $C^{\prime}$ respectively. Let the cost $C^{\prime}(j, i)$ of the link $(j, i)$ in graph $G^{\prime}$ for each link $(i, j)$ along the shortest path $p$ is set to $-C(j, i)$ (negative cost). Remove the directed links $(i, j)$ along the shortest path $p$ in $G^{\prime}$. Compute a shortest path $p^{\prime}$ from $s$ to $t$ in the modified graph $G^{\prime}$. It is important to note that the 
modified graph has edges with negative costs. Remove the interlacing edges on paths $p$ and $p^{\prime}$ to get a pair of least cost link disjoint paths. The paths computed by Suurballe's algorithm need not be SRLG diverse.

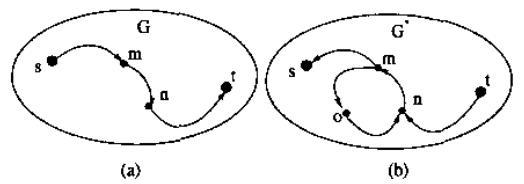

Fig. 1. (a) Graph G with the shortest path $(s \rightarrow m \rightarrow n \rightarrow t)$. (b) Modified graph $G^{\prime}$ with the edge costs along the shortest path $(s \rightarrow m \rightarrow n \rightarrow t)$ negated.

Theorem III.1: The modified graph $G^{\prime}$ in Suurballe's algorithm has no negative cost cycle.

Proof: Consider the graph $G$ shown in the Fig. 1(a) where $s \rightarrow m \rightarrow n \rightarrow t$ is the shortest path between node pair $(s, t)$ and $m, n$ are intermediate nodes. The modified graph $G^{\prime}$ is shown in the Fig. 1(b). Let $C^{\prime}(n \rightarrow m)$ denote the cost of the sum of all the edges along the path segment $n \rightarrow m$ of the path $p$. Suppose for contradiction that $G^{\prime}$ has a negative cost cycle $(m \rightarrow o \rightarrow n \rightarrow m)$. Therefore,

$$
\begin{aligned}
C^{\prime}(n \rightarrow m)+C^{\prime}(m \rightarrow o \rightarrow n) & <0 \\
-C(n \rightarrow m)+C(m \rightarrow o \rightarrow n) & <0 \\
C(m \rightarrow o \rightarrow n) & <C(n \rightarrow m)
\end{aligned}
$$

It implies the path $(s \rightarrow m \rightarrow o \rightarrow n \rightarrow t)$ is shorter than path $(s \rightarrow m \rightarrow n \rightarrow t)$ in the original graph $G$. A contradiction, since we assumed that $(s \rightarrow m \rightarrow n \rightarrow t)$ is the shortest path. Therefore $G^{\prime}$ has no negative cost cycle.

Therefore by Theorem III.1 Suurballe's algorithm cannot be used in the iterative heuristic for computing the least cost diverse paths. Since using any other path than the shortest path as the seed path in the iterative procedure may result in negative cycle in the modified graph. Then the shortest path cannot be found in the modified graph. In this work we present a modified Suurballe's heuristic (MSH). We propose an iterative heuristic (IMSH) which uses the MSH to compute the least cost SRLG diverse path pair for a connection. We also present an $1 / 2$ cost-improvement optimality verification criterion for our proposed IMSH under the dedicated protection. In the next section we describe the modified Suurballe's heuristic and IMSH to compute the least cost SRLG diverse paths.

\section{OUR ITERATIVE APPROACH}

Given an undirected weighted graph $G=(V, E)$ with SRLG constraints $R$ and a node pair $(s, t)$, the least cost SRLG diverse routing (LC-SDR) problem is to find a pair of SRLG diverse routes between $s$ and $t$.

\section{A. Modified Suurballe's Heuristic (MSH)}

Let us first describe the modified Suurballe's heuristic to compute the SRLG diverse path pair. The outline of the modified Suurballe's heuristic is given in the Algorithm 1. The input to the heuristic is a directed graph $G$ with a set of SRLG $R$ and a cost function $C$ of the edges. Given the seed path $p$ between the source $s$ and destination $t$ the heuristic returns a pair of SRLG diverse paths if such an SRLG diverse path pair can be computed, else it retums NULL. We call this modified Suurballe's algorithm a heuristic since it does not guarantee that it will always return a pair of SRLG diverse routes.

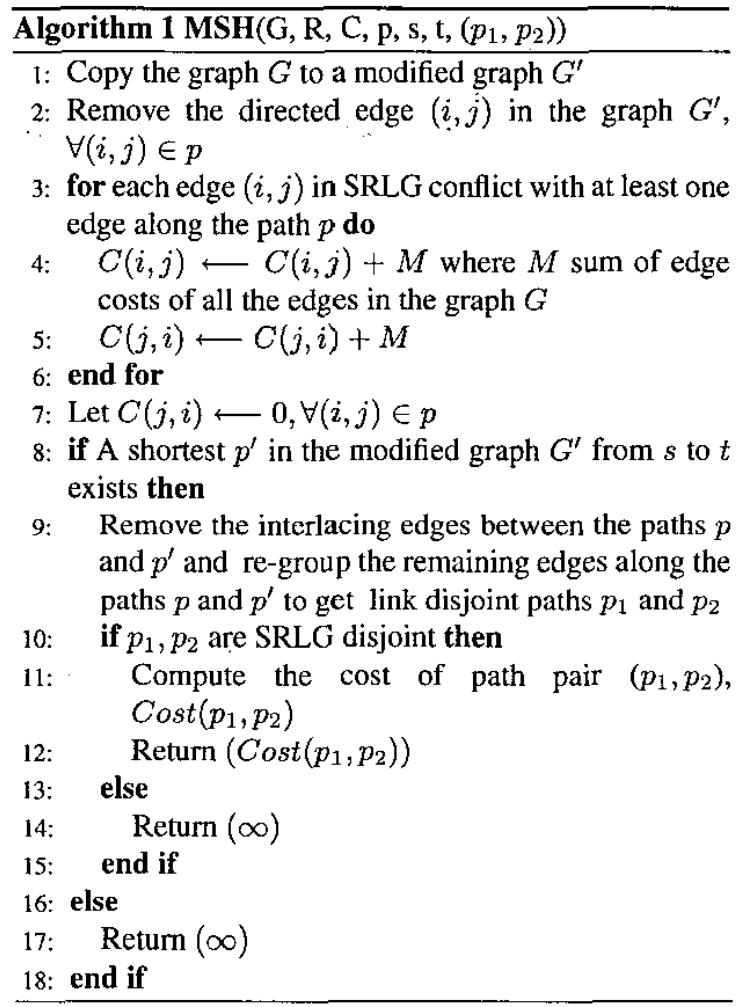

Let us discuss the working of the modified Suurballe's heuristic. Steps 1-7 compute a modified graph $G^{\prime}$ of the input graph $G$ based on the seed path $p$. In steps 3-6, the cost of the links that are in SRLG conflict with the links along the path $p$ is increased by $M$ (sum of the costs of all the links in the network) to give lower priority to these conflict-links while computing SRLG disjoint path in the modified graph. The rest of the heuristic (steps 8-18) is similar to the Suurballe's algorithm except in step 10 the disjointedness of the path $p_{1}$ and $p_{2}$ is verified. Since the edges in the modified graph $G^{\prime}$ have non-negative costs, Dijkstra's algorithm can be used to compute the shortest path in modified graph $G^{\prime}$ in Step 8. We know that the time complexity of Dijkstra's algorithm 
is $O(m+n \log n)$ where $n$ is the number of nodes and $m$ is the number of links/edges in the network. Step 9 takes $O(n)$ time, since the shortest path has at most $n$ edges. The Step 10 takes $O(n+r)$ time where $r$ is the number of shared risk link groups in the network. Therefore the time complexity of the modified Suurballe's heuristic is $O(m+r+n \log n)$. The cost of the path pair computed by the modified Suurballe's heuristic is at most the cost of the path pair computed by the two-step approach. Theorem IV.1 proves this essential fact.

Theorem IV.1: Given a graph $G=(V, E)$, the cost function $C$ for its edge set $E$, a seed path $p$ from source $s$ to destination $d$. If $C_{M S}, C_{T S}$ be the costs of the SRLG diverse path pair computed by modified Suurballe's heuristic and the two-step approach respectively then $C_{M S} \leq C_{T S}$.

Proof: Let $G^{\prime}, G^{\prime \prime}$ be the modified graphs of modified Suurballe's heuristic and two-step approach respectively. Let $p^{\prime}$ and $p^{\prime \prime}$ be the shortest paths from $s$ to $t$ computed in the modified graphs of the modified Suurballe's heuristic and two-step approach respectively. We know that in $G^{\prime}$ all the directional edges $(i, j)$ along the seed path $p$ are removed and the cost link $(j, i)$ for all the links $(i, j)$ along the seed path $p$ is zero. And we also know that links $(i, j)$ and $(j, i)$ for all the links $(i, j)$ along the seed path $p$ are removed in the graph $G^{\prime \prime}$. There exists two cases for path $p^{\prime}$.

Case 1: $p^{\prime}$ does not use any of the links $(j, i)$ whose cost is zero. Then the path pair computed by modified Suurballe's heuristic is $\left(p, p^{\prime}\right)$ and its cost is $C_{M S}=$ $\operatorname{Cost}(p)+\operatorname{Cost}\left(p^{\prime}\right)$ where $\operatorname{Cost}(p)$ and $\operatorname{Cost}\left(p^{\prime}\right)$ are the costs of the paths $p$ and $p^{\prime}$ respectively. It is easy to see that $p^{\prime}$ is also the shortest path from $s$ to $t$ in the graph $G^{\prime \prime}$ i.e., $p^{\prime}=p^{\prime \prime}$. Therefore $C_{T S}=\operatorname{Cost}(p)+\operatorname{Cost}\left(p^{\prime}\right)=$ $C_{M S}$.

Case 2: $p^{\prime}$ uses some link $(j, i)$ whose cost is zero. Then the cost of the path pair computed by modified Suurballe's heuristic, $C_{M S} \leq \operatorname{Cost}(p)+\operatorname{Cost}\left(p^{\prime}\right)-$ $C(i, j)$ where $C(i, j)$ is the cost of the link $(i, j)$ in the original graph $G$. Since the modified Suurballe's heuristic removes the interlacing link $(i, j)$ and computes the path pair using the remaining links along the path $p$ and $p^{\prime}$. The cost of the path pair computed by two-step approach is $C_{T S}=\operatorname{Cost}(p)+\operatorname{Cost}\left(p^{n}\right)$. By the construction of graphs $G^{\prime}$ and $G^{\prime \prime}, \operatorname{Cost}\left(p^{\prime}\right) \leq \operatorname{Cost}\left(p^{\prime \prime}\right)$. Then $C_{M S} \leq C_{T S}$. Hence proved.

\section{B. Iterative Modified Suurballe's Heuristic (IMSH)}

Let us now describe the iterative heuristic for computing the least cost SRLG diverse paths. The IMSH iteratively executes the modified Suurballe's heuristic using $i^{t h}$ shortest path as the seed path in each iteration. In the $i^{\text {th }}$ iteration, the heuristic computes the $i^{\text {th }}$ shortest path from $s$ to $t$ using Yen's algorithm [15]. The heuristic computes the SRLG path pair using the modified Suurballe's heuristic with $i^{t h}$ shortest path as the seed path. Let $\left(p_{i}^{\prime}, p_{i}^{\prime \prime}\right)$ be the SRLG diverse path pair computed and its cost be $\operatorname{Cost}\left(p_{i}^{\prime}, p_{i}^{\prime \prime}\right)$. If SRLG diverse path pair could not be computed, the heuristic continues with the next iteration. If IMSH heuristic is allowed to run until all the shortest paths are explored it will eventually stop and give the optimal solution. We restrict the maximum number of times the heuristic can iterate by a pre-determined upper bound 'MAX-ITERATIONS'. The IMSH if allowed to run until all the shortest paths are explored it is an algorithm. Since the number of possible shortest paths between any node pair in a networks can be exponential we restrict the number of iterations and so is IMSH a heuristic.

Let us now present the optimality verification criterion of the proposed IMSH. Let $P P_{c u r-o p t}$ be the current optimal SRLG diverse path pair found and its cost be $C_{c u r-o p t}$. In the $i^{t h}$ iteration, let $\operatorname{Cost}\left(p_{i}\right)$ be the cost of the shortest path computed using Yen's algorithm [15]. Let $\left(p_{i}^{\prime}, p_{i}^{\prime \prime}\right)$ be the SRLG diverse path pair computed using modified Suurballe's heuristic, if such a path pair exists. Let $\left(p_{i}^{\prime}, p_{i}^{\prime \prime}\right)$ be more optimal than the current optimal $P P_{c u r-o p t}$, i.e.,

$$
\operatorname{Cost}\left(p_{i}^{\prime}\right)+\operatorname{Cost}\left(p_{i}^{\prime \prime}\right)<C_{c u r-o p t}
$$

Now $\operatorname{Cost}\left(p_{i}^{\prime}\right), \operatorname{Cost}\left(p_{i}^{\prime \prime}\right) \geq \operatorname{Cost}\left(p_{i}\right)$. Since, without loss of generality, if $\operatorname{Cost}\left(p_{i}^{\prime}\right)<\operatorname{Cost}\left(p_{i}\right)$ the optimal SRLG path pair must have already been computed using $p_{i}^{\prime}$ as the seed path. Therefore,

$$
2 \operatorname{Cost}\left(p_{i}\right) \leq \operatorname{Cost}\left(p_{i}^{\prime}\right)+\operatorname{Cost}\left(p_{i}^{\prime \prime}\right)
$$

From Eq. 1 and 2 we get,

$$
\begin{aligned}
2 \operatorname{Cost}\left(p_{i}\right) & <C_{\text {cur-opt }} \\
\operatorname{Cost}\left(p_{i}\right) & <C_{\text {cur-opt }} / 2
\end{aligned}
$$

Therefore if the cost of the current seed path in the $i^{\text {th }}$ iteration is greater than or equal to $C_{c u r-o p t} / 2$, then the optimal SRLG diverse path pair is $P P_{c u r-o p t}$.

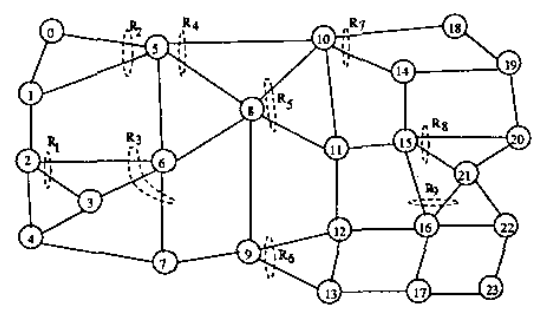

Fig. 2. 24-node 43-link physical network with SRLGs 

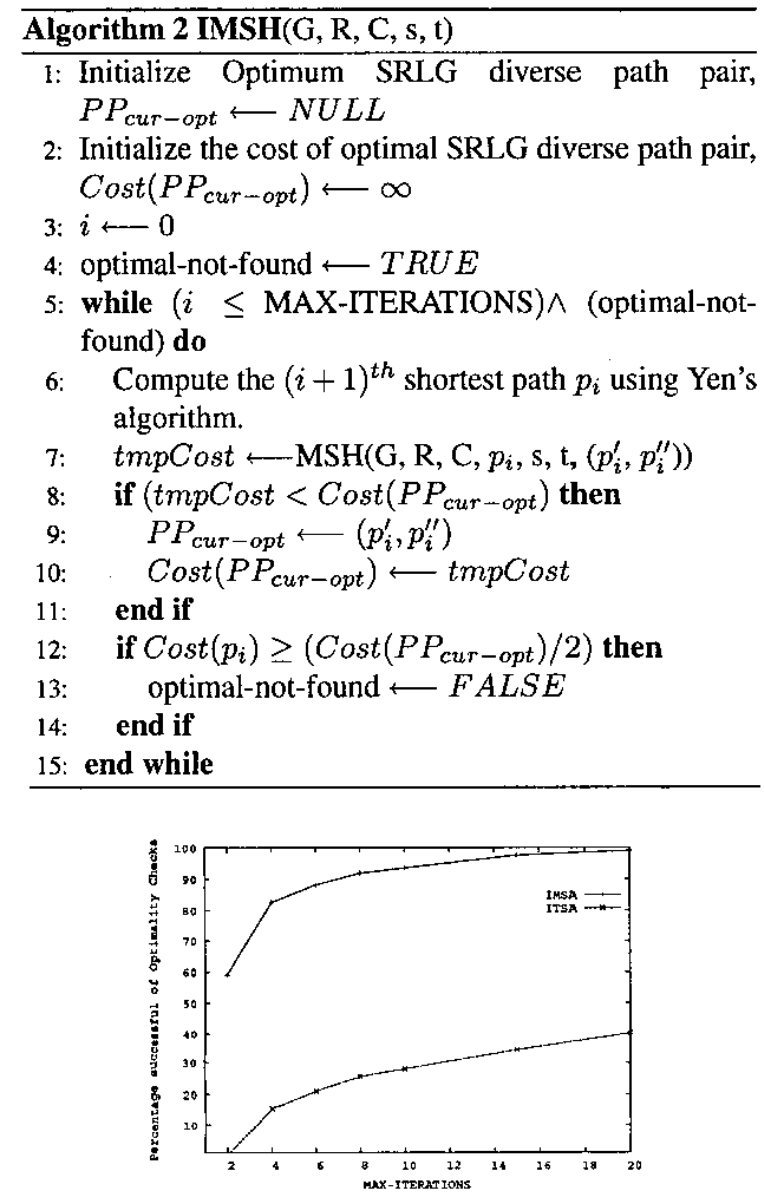

Fig. 3. Percentage of successful optimality checks using ITSH and IMSH on 24-node network.

\section{EXPERIMENTAL DESIGN}

We conducted experiments on 24-node network (shown in Fig.2) and 14-node NSF network. We assume that the number of wavelengths on each fiber link in both the networks is 16 . We simulated dynamic traffic with calls arriving into the network having Poisson distribution with mean arrival rate $\lambda$. The connections have exponential holding time with mean $1 / \mu$. The load on the network is measured as $\lambda / \mu$. The number of calls simulated in the network during each simulation run are 10,000 and 100,000 for 24-node network and NSF network respectively. We implemented both the heuristics ITSH and IMSH. The performance is measured in terms of blocking probability, percentage of optimality checks, average number of iterations per call and the average time taken to compute the SRLG diverse path pair. The blocking probability is the ratio of the number of blocked calls to the total number of calls simulated. The percentage of optimality checks is the percentage of calls

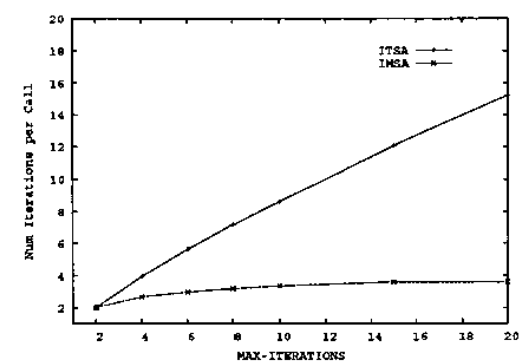

Fig. 4. Average number of iterations per call using ITSH and IMSH on 24-node network.

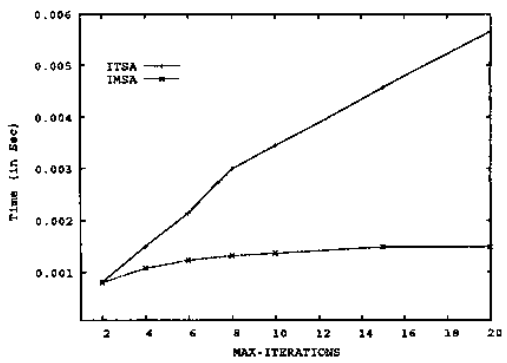

Fig. 5. Average computation time of least cost SRLG diverse path pair using ITSH and IMSH in 24-node network.

for which the optimality (least cost) of the SRLG diverse path pair is verified. The average number of iterations is the average of the total number of iterations executed by the heuristics (ITSH or IMSH) for computing the diverse path pair for the number of calls simulated. The average time taken (in seconds) for computing the SRLG diverse paths for a call. The experiments are run on a unloaded 1 GHz AMD Athlon machine with 256 MB RAM.

The blocking probability for both the heuristics is almost same and therefore the graph is not shown. Fig. 3, Fig. 4 and Fig. 5 show the percentage of successful optimality checks, average number of iterations per call and average computation time respectively for varying upper bound on the number of iterations of the heuristics (ITSH and IMSH). The results also show that IMSH verifies the optimality of SRLG diverse path pair for $99.08 \%$ of the connections when the upper bound on the number of iterations is 20 . While ITSH could only verify the optimality of $40.38 \%$ of connections. With the increasing limit on the number of iterations, the average number of iterations per connection for the ITSH for computing and verifying the optimality of the SRLG diverse path pair also increases linearly. In contrast the number of iterations per connection for the IMSH is almost constant.

Fig. 6 and Fig. 7 show the percentage of optimality verifications and the average number of iterations per call for the ITSH and IMSH on NSF network topology. The 


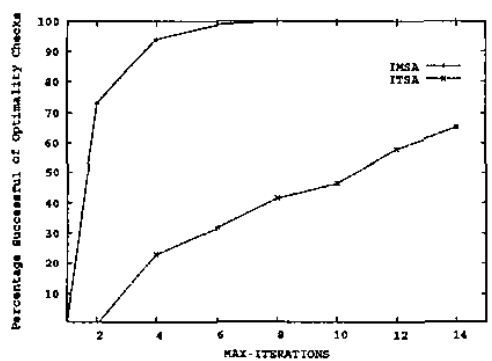

Fig. 6. Percentage of successful optimality checks using ITSH and IMSH on NSF network.

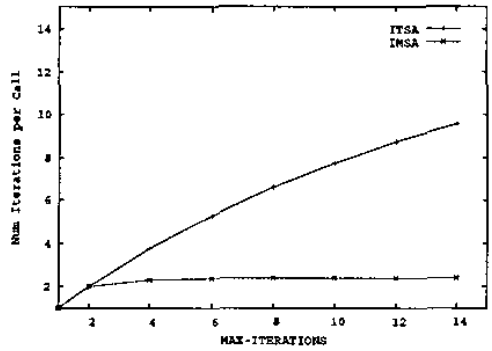

Fig. 7. Average number of iterations per call using ITSH and IMSH on NSF network

percentage of optimality checks for IMSH grows quickly when the upper bound on the number of iterations of IMSH is increased from 2 to 6 and reaches $100 \%$ when the upper bound is 14 . The percentage of optimality checks for ITSH grows but relatively slowly when compared to IMSH. The average number of iterations per call before stopping for the ITSH increases almost linearly with the increasing upper bound on the number of iterations of the ITSH. In contrast the average number of iterations for IMSH is almost constant. The blocking probabilities of ITSH and IMSH are almost similar (graph not shown). The results from the 24-node and NSF networks show that IMSH computes the optimal solutions more efficiently than the ITSH.

\section{CONCLUSIONS}

In this work we studied the problem of SRLG diverse routing for protection. The problem of diverse routing has been extensively studied in the literature in the context of link disjointedness and SRLG diversity under shared protection. We presented a modified Suurballe's heuristic for computing SRLG diverse routes. We presented an iterative heuristic (IMSH) for computing least cost SRLG diverse routes using modified Suurballe's heuristic. Based on the independent cost structure of working and protection paths in dedicated protection we presented a 1/2-cost-improvement optimality verification criterion. The experimental results show that both ITSH and the proposed IMSH have almost the same blocking probability for 24-node and 14-node NSF networks. The proposed IMSH computes the solution more efficiently than ITSH and verifies the optimality of almost all the solutions.

\section{REFERENCES}

[1] J. Q. Hu, "Diverse Routing in Optical Mesh Networks", IEEE Transactions on Communications, Vol. 51, No. 3, pp.489-494 2003.

[2] Pin-Han Ho, "State-of-the-Art Progress in Developing Survivable Routing in Optical Metropolitan Area Networks," IEEE Communications Surveys and Tutorials.

[3] J. Strand, A. L. Chiu, and R. Tkach, "Issues for Routing in the Optical Layer," IEEE Communications Magazine vol. 39, issue. 2 , pp. 81-87, Feb 2001

[4] J. W. Suurballe and R. E. Tarjan, "A Quick Method for Finding Shortest pair of Disjoint Paths," Networks, vol. 14, no. 2, pp. 325$336,1984$.

[5] J. Tapolcai, P. Laborczi, P-H. Ho, T. Cinkler, A. Recski, H. T. Mouftah, "Approaches for Solving Asymmetrically Weighted Optimal or Near-Optimal Disjoint Path-Pair for the Survivable Optical Networks," Third International Workshop on Design of Reliable Communication Networks (DRCN '01), Oct. 2001 .

[6] H. Zang, C. (Sam) Ou, and B. Mukherjee, "Path-Protection Routing and Wavelength Assignment (RWA) in WDM Mesh Networks Under Duct Layer Constraints," IEEE/ACM Transactions on Networking, vol. 11, no. 2, April 2003.

[7] P-H. Ho, J. Tapolcai, and H. T. Mouftah, "Diverse Routing for Shared Protection in Survivable Optical Networks," in Proc. IEEE Global Telecommunications Conference (GLOBECOM '03), vol. 5, pp. 2519-2523, December 2003.

[8] J-F. Labourdette, "Shared Mesh Restoration in Optical Networks," in Proc. Optical Fiber Communication (OFC '04), TuP3, Los Angeles, CA, Feburary 2004.

[9] P-H. Ho, J. Tapolcai, and H. T. Mouftah, "On Achieving Optimal Survivable Routing for Shared Protection in Survivable NextGeneration Internet," IEEE Transaction on Reliability (accepted).

[10] G. Li, R. Doverspike, and C. Kalmanel, "Fiber Span Failure Protection in Mesh Optical Networks", in OptiComm 2001: Optical Networking and Communications, Proc. SPIE, Vol. 4599 pp. 130-142, 2001

[11] S. Z. Shaikh, "Span-Disjoint Paths for Physical Diversity in Networks", in Proc. of IEEE Symposium on Computers and Communications, pp. 127-133, 1995

[12] L. Shen, X. Yang and B. Ramamurthy, "Shared Risk Link Group (SRLG)-Diverse Path Provisioning under Hybrid Service Level Agreements in Wavelength-Routed Optical Mesh Networks: Formulation and Solution Approaches," SPIE OptiComm 2003, Dallas, TX, Oct. 2003

[13] P. Datta, A. K. Somani, "Diverse Routing for Shared Risk Resource Group (SRRG's) in WDM Optical Networks,".

[14] B. Korte, L. Lovasz, H. J. Promel, A. Schrijver, Paths, Flows, and VLSI-Layout, Algorithms and Combinatorics, Springer-Verlag, 1990.

[15] J. Y. Yen, "Finding the k shortest loop less paths in a network," Management Science vol. 17, pp. 712-716, 1971. 